\title{
The Role of Family Physician in Palliative Care
}

\author{
Saima Perwaiz lqbal ${ }^{1 *}$ \\ ${ }^{1}$ Department of Family Medicine, Shifa College of Medicine, Shifa Tameer-e-Millat University, Islamabad, Pakistan
}

\begin{abstract}
The following article deals with the role of Family Physician (FP) in Palliative Care Medicine (PCM). The author will discuss how a Family Physician should deal with dying patients and their aggrieved families within the context of Pakistan. It is hoped that after reading through the article, readers will be able to appreciate the unmet needs of dying patients and their families, identify knowledge gaps and assess the need to enhance communication/counseling skills and coping strategies for the improvement of care for dying patient.
\end{abstract}

Keywords: Palliative care, family medicine, family physician.

\section{INTRODUCTION}

World Health Organization (WHO) defines the purpose of palliative care to improve the quality of life of patients suffering from life threatening illnesses by preventing and relieving their suffering through identification and assessment of their pain and other biological, psychosocial and spiritual problems. Palliative care is stated to be "an ethical responsibility of health care systems" globally [1]. Palliation cannot be done in isolation. Multiple facets of care need to be examined and evaluated and the patient should be treated accordingly. Therefore, the delivery of palliative care requires a team based approach which involves health care professionals like doctors and nurses as well as allied health care specialists including physiotherapists, nutritionists, psychologists and occupational therapists. Apart from health care specialists and allied specialties the role of family, friends and spiritual healers cannot be ignored. Especially in a country like Pakistan which has a collectivistic culture, the family unit is considered to be of prime importance especially at the time of death.

Life and death are regarded as natural processes and in Muslim majority countries like Pakistan, most of the population believe that time of death is preordained. However, there is a cultural reluctance of the general Pakistani population to discuss about death and dying as it is considered ominous and therefore avoided [2]. Subsequently, Pakistani health care professionals also avoid discussing death and dying with their patients. Quite often it is the family member who instructs physicians not to disclose the diagnosis and prognosis to the patient in an effort to protect them from bad news and depression. This is called the "conspiracy of silence" and one can debate as to its ethical implications [3].

*Corresponding Author: Saima Perwaiz Iqbal, Department of Family Medicine, Shifa College of Medicine, Shifa Tameer-e-Millat University, Islamabad,Pakistan; Email: saima.scm@stmu.edu.pk

Received: October 28, 2019; Revised: December 31, 2019; Accepted: December 31, 2019 DOI: https://doi.org/10.37184/lnjpc.2707-3521.1.6

\section{ROLE OF FAMILY PHYSICIAN IN \\ PALLIATIVE CARE MEDICINE}

A trained family physician is the best person to coordinate care for a patient requiring palliative care in the context of Pakistan. This is because most people would appreciate a trusted family doctor to whom they go to for their everyday problems. Family physicians are spread out in the community and can provide good quality care if trained to do so at the patient's door step. The following steps are proposed to help family physicians become accustomed to the idea of providing palliative care to the patients that they treat in the community.

\section{Identifying the Dying Patient:}

Palliative care deals mostly with patients suffering from cancers plus non-cancer illnesses. When diagnosed with cancer, patients or their families may ask the physician, how long they have got to live. Being able to provide an accurate prognosis for survival is a core clinical skill of the physician and family physicians need to enhance this ability despite there being an element of uncertainty in the process. Various tools have been developed in this regard to determine prognosis of a dying patient depending upon current functional status, ability for self care, requirement of institutional care etc. $[4,5]$.

However, care must be taken when applying these tools as prognostic accuracy is dependent upon patient population, setting and timeline [6]. Prognostication is a complex process with several interdependent factors. It becomes more probabilistic in patients suffering from a non-cancer illness like heart failure and respiratory failure [7]. Therefore it is important to convey information to patients and their families with sensitivity, consideration and a certain degree of uncertainty.

If a doctor suspects that a patient may be entering into the last few days of life he/she should gather information on the patients physiological, psychological, social and spiritual needs. The family should be involved in respecting the dying person's wishes and future care. Look for signs of terminal agitation, Cheyne-Stokes 
breathing, altered level of consciousness, mottled skin, "death rattle" which is noisy breathing caused by pooled secretions, progressive weight loss, increasing fatigue and loss of appetite as these are prognostic indicators of imminent death. Any improvement in signs could indicate that the patient is recovering or improving. Any investigations should be avoided which are unlikely to change the management plan at the end of life.

\section{Communicating with Patient and Family:}

Palliation starts once the patient has been diagnosed with a life threatening illness. Breaking this bad news requires a skill and compassion for it to be delivered effectively to the patient and his/her family. Bad news has the potential to cause frustration, sadness, disappointment and hostility in patients and families receiving it. Doctors can also feel inadequate, apprehensive, confused and upset if they have to deliver it. All trained family physicians need to equip themselves with this skill as good communication is an inherent part of the doctorpatient relationship.

Apart from breaking bad news, doctors should be skilled in communicating accurate information about prognosis to the patient and family members. Shared decision making is the standard to uphold. The plan should include taking into account the dying person's wishes, preferred setting of care, preferences for symptom management and addressing any underlying concerns and fears. Unfortunately in Pakistan doctors are neither adequately trained nor confident about the process of delivering bad news to their patients $[8,9]$. On the other hand, patients in our country expect their respective doctor and/or health care provider to be honest and upfront about any bad news given to them [10].

How can family physicians equip themselves with the skill of breaking bad news if they have not encountered formal training sessions in their undergraduate and postgraduate teaching? First step is to read about it. Various articles and books are available which can help physicians to build up this skill. A commonly used model is the SPIKES protocol [11]. YouTube videos are also available by which viewer can observe the body language, tone of voice, use of words and appropriate use of silences. Then, recall instances as a medical student or doctor or as a patient or a relative in which you have observed, witnessed or experienced an encounter in which bad news was delivered. Reflect on what went well and what didn't go so well. Think how it could have been improved. Be cognizant of the emotions and reactions of patients. Think how the physician can be empathetic and professional at the same time. Recognize the fact that delivering bad news does take an emotional toll on the doctor as well. Develop a coping strategy or mechanism to prevent burnout. Some doctors take to writing, some indulge in exercise, and some may talk to a friend. These "housekeeping" methods will keep the physician mentally and emotionally strong to carry out his/her duties despite dealing with pressures of breaking bad news and dealing with dying patients [12].

Dealing with Common Problems of the Dying Patient: Once diagnosed with a terminal illness, the family physician should be aware of the common problems that can occur with the patient. Pain, nausea/ vomiting, breathlessness and constipation are some of the most frequently encountered symptoms in palliative care [13]. The family physician should ideally be trained in all these aspects. Familiarity with the WHO pain ladder is essential for pain management [14]. To manage nausea/ vomiting the doctor needs to be mindful of the underlying cause and the physiological pathway involved for better management and choice of anti-emetic. Managing breathlessness requires the family physician to be aware of the underlying cause like anxiety, effusions, heart failure, and then treat accordingly. If the patient is suffering from constipation then again look for the underlying cause related to the disease, drugs, fluid depletion, intestinal obstruction, metabolic disturbance and so on.

Maintaining nutrition and hydration at the end of life is also imperative for the family physician to review. Patients are entitled to food and hydration of adequate amount and appropriate quality. Caretakers and the patient's family need to be guided as to how to encourage and support the patient to eat and drink to prevent malnutrition and dehydration. If a patient refuses to eat or drink address any underlying cause like inability to swallow depression or mouth ulcers. With the help of a dietician, the family physician may assess the need for providing clinically assisted nutrition like nasogastric feeding, intravenous feeding or insertion of percutaneous endoscopic gastrostomy (PEG).

\section{Coordinating with Other Health Care Services:}

Palliative care patients may require additional services like nutrition care, physiotherapy, psychotherapy and spiritual care. Unfortunately in Pakistan the health care system is not that well established to facilitate such services smoothly. In this case a trained family physician is the best person to advise and coordinate such services and guide patients and their families accordingly. Family physicians in Pakistan need to network with nurses and these allied health services to provide comfort care for their dying patients. We would hope that health policy makers would focus on this unmet need of palliative care and devise a strategy for its deliverance and implementation by bringing together different specialties on board. Educational organizations can play a role by educating future doctors and nurses by implementing palliative care in their respective curricula.

\section{Allowing a Comfortable Death:}

Death is a certainty that all of us living in a Muslim country are aware of and being Muslims we believe that a time of death is pre-ordained. That does not mean that death has to be painful or unnecessarily prolonged. In the late 
stages of death patients may fall into an altered state of consciousness, have an altered respiratory pattern, mottled extremities and because of pooled oral secretions may demonstrate what is known as "death rattle". The family physician's role would be to identify and provide comfort care to the patient. The families also need to be counseled about remaining calm and controlling their own distress in the patient's presence. They should not push for any tests or treatments which would be futile. Advise the family and caretakers to put the patient into a comfortable position. Let other family members, friends and even children visit the dying patient. If experiencing any symptoms like pain then treat with medications. If patient is not drinking you may wet the mouth with a sponge or give ice chips. If patient is hot, apply a cool cloth on his forehead. If patient complains of blurred vision, keep a light on. Ask family members to maintain some amount of physical contact with the patient or hold hands. Talk calmly and softly to the patient even if he/she does not respond. If patient is drowsy allow him to sleep. Religious and cultural practices like recitation from the Holy Quran and/or Suras may prove to be beneficial to both patients and their families.

\section{After Death Care of Family:}

Once the patient passes away there is a feeling of loss and grief. Family physicians need to be aware of the stages of bereavement and provide emotional and psychological support to those left behind [15]. Religious rituals in our country in which friends and family gather around the family of the deceased and funeral rites provide some sort of healing and acceptance of the death. Family doctors should be vigilant about family members of the deceased being prone to depression beyond the bereavement process. The medical certificate of the cause of death should be issued within one to two working days by the family doctor.

\section{MEETING THE NEED THROUGH EDUCATION}

Caring for those at the end of life and after death is an important responsibility. As palliative medicine is still an under developed specialty in Pakistan, there is more need for it to be taught in our undergraduate medical and nursing curriculum $[16,17]$. With so much information overload, it is suggested that where there are established departments of Family Medicine or Internal Medicine it can be introduced to give students a concept that dying patients also deserve care. There are limited palliative care specialists in Pakistan and most have completed their specialist training from abroad. With establishment of palliative medicine as a specialty it will pave the way to deliver an unmet need for our dying patients.
Palliative care cannot be delivered by the palliative care specialist in isolation. There needs to be coordination with the family physician, home care nurse, physiotherapist, psychologist and a spiritual leader that the patient is comfortable with. Only then can we truly fulfill our population's need for palliative care at the end of life.

\section{REFERENCES}

1. Integrating palliative care and symptom relief into primary health care. A WHO guide for planners, implementers and managers. ISBN 978-92-4-151447-7

2. Khan RI. Palliative care in Pakistan. Indian J Med Ethics 2017; 2(1): 37-43.

3. Qidwai W. Care of the dying patient: a neglected issue in Pakistani context. J Coll Phys Surg Pak 2012, 22(3): 133-4.

4. Mendis R, Soo WK, Zannino D, Michael N, Spruyt O. Multidisciplinary prognostication using the palliative prognostic score in an Australian cancer center. Palliat Care 2015; 9: 7-14.

5. Prognostic Indicator Guidance to aid identification of adult patients with advanced disease, in the last months/year of life, which are in need of supportive and palliative care. The gold standards framework. 4th ed. United Kingdom: Royal College of General Practitioners Available at: https://geri-em.com/wp-content/ uploads/2013/05/Prognostic-Indicator-Guide.pdf. [Accessed on: December 16, 2019].

6. Chu C, White N, Stone P. Prognostication in palliative care. Clin Med 2019; 19(4): 306-10.

7. The National Hospice Organization. Medical Guidelines for Determining Prognosis in Selected Non-Cancer Diseases. Hosp J 1996; 11(2): 47-63.

8. Bukhari SAH. "Breaking bad news" Current practices in hospitals. Ortho Rheum Open Access J 2018; 11(2): 555806.

9. Baig L, Tanzil S, Ali SK, Sheikh S, Jamali S, Khan M. Breaking bad news: a contextual model for Pakistan. Pak J Med Sci 2018; 34(6): 1336-40.

10. Ishaque S, Saleem T, Khawaja FB, Qidwai W. Breaking bad news: exploring patient's perspective and expectations J Pak Med Assoc 2010; 60(5): 407-11.

11. Baile WF, Buckman R, Lenzi R, Glober G, Beale EA, Kudelka AP. SPIKES-a six-step protocol for delivering bad news: application to the patient with cancer. Oncologist 2000; 5(4): 302-11.

12. Warriner D. The inner consultation. BMJ Clin Res 2008; 337 a1574.

13. World Health Organization. Essential medicines in palliative care. Executive Summary. International Association for Hospice and Palliative Care (IAHPC). Available at: https://www.who. int/selection medicines/committees/expert/19/applications/ PalliativeCare_8_A_R.pdf [Accessed on: December 16, 2019].

14. WHO's cancer pain ladder for adults. Available at: https://www. who.int/cancer/palliative/painladder/en/ [Accessed on: December 16, 2019].

15. Ross EK, Kessler d. On grief and grieving: finding the meaning of grief through the five stages of loss. Simon \& Schuster 2005.

16. Punjwani R, Khatoon A, Dias JM, Siddiqui DF, Bana S, Baig-Ansari $\mathrm{N}$. Palliative care in nursing-where are we in Pakistan. J Palliat Care Med 2015; S5: S5-002.

17. Abbas SQ, Muhammad SR, Mubeen SM, Abbas SZ. Awareness of palliative medicine among Pakistani doctors: a survey. J Pak Med Assoc 2004; 54: 195 